\title{
Privacy in China: An acculturation perspective
}

\author{
Ho Manh Tung \\ Ritsumeikan Asia Pacific University \\ Верри, Oita, Japan
}

November 18, 2020

Privacy trust is an important global issue in ensuring data quality. When people do not volunteer data or volunteer as much false data as possible, it will become a problem for data collectors and algorithm designers. This is a concern for private companies, social organizations, and governments alike. Even a big government such as the Chinese would not be exempt from this problem. Although we live in a hyperconnected, globalized world, the truth is both privacy and trust are concepts that have deep, entrenched cultural nuances. This essay reviews some prominent findings and discussions on privacy, trust, and data collection ethics in China to understand the influence of Confucianism on the perception and governance of algorithmic design.

According to Han (2018), research on privacy issues in China can be divided into three camps: privacy and governmental surveillance; legal frameworks around what constitutes private information; profit-driven collection and exploitation of personal data. I would add to this list the research area of public behaviors and perception regarding data privacy (Wang and $\mathrm{Yu}, 2015$; Wang, Balnaves, \& Sandner, 2020).

On privacy and government surveillance, it is important to state that there is no concept in Chinese philosophies that directly translates to privacy as a moral right of freedom from 
surveillance and state interference, as commonly conceived in the West (Whitman, 1985). Furthermore, this particular way of understanding privacy in the West is not innate and immutable; as with any other discourses, the Western concept of privacy is a product of culture and history (Whitman, 1985). In China, given the context of globalization and commodification of many cultural aspects $(\mathrm{Li}, 2017)$, one can argue that as an imported concept, privacy is going through a process of acculturation in China ((Vuong \& Napier, 2015; Vuong et al., 2018; 2020).

To elaborate, the traditional Confucian and Taoist values and expectations are coming into conflict with the Western norms of privacy. For example, Ma (2019) argues privacy is an unmapped concept, and it is an open debate that whether within the traditional Confucian thoughts, an individual must be subordinated to the group. How the Chinese public perceives this matter holds enormous influence over what constitutes private information greatly and, thus, surveillance practice. Then in the research area of public behaviors and perception regarding data privacy, Wang and Yu (2015) find socio-demographic factors shape privacy perceptions: older people take less precaution to protect their data, and more educated people take more precaution, and people have more suspicions towards the private sectors than the governments, social associations, and public services. Meanwhile, Wang, Balnaves, and Sandner (2020) find the Confucian concept of guanxi (social relationship) is key to understand the perception of privacy among young and rural women in China: privacy is the level of control over the identity they present on the technological platforms such as WeChat, Weibo, etc.

It is interesting that in a country with such a big and strong state apparatus like China, we find the legal frameworks around and the definition of private information are driven by the bottom-up phenomenon of public behaviors regarding privacy. An interesting phenomenon in China is the Human Flesh Search (HFS) phenomenon, which has shaped the development of 
privacy law in China. The HFS is an online bottom-up collaborative effort of netizens to search and provide first-hand information to online forums about individuals and companies that the Internet communities deem suspicious or worthy of condemnation, even harassment. Notably, this phenomenon does not only confine to an individual citizen. Many Chinese people found unfavorable first-hand information about Chinese companies listed in stock markets, which contradict the companies' disclosed information. There have also been cases where HFS targeted corrupt government officials. These examples seem to diverge from the heavy-handed, strict rule of Internet governance in China.

Han (2018) argues this phenomenon is a public online collaborative response to situations where institutionalized media are perceived to not fulfilled their role. The divergence is merely an appearance, as the government's "tolerance" toward the HLS phenomenon highlights, according to Han (2018), both the "flexibility and populism" embedded in the Chinese governing technique. Using the example of privacy law in China, Han (2018) shows. However, privacy has an ambiguous status within the Chinese legal framework; the high-profile HFS cases have triggered multiple lawsuits and, consequently, interpretations and further developments of the privacy regulations to define the contested boundaries of online search. Invoking the framework of "flexibility and populism" proposed by Liebman (2011), Han (2018) shows the development of privacy law in China is consistent with the historical Chinese governing technique that seeks to maintain political and social hierarchy as well as provide legitimacy and authority to the government.

On the issue of legitimacy and authority of the government, as Whitman (1985) has observed, the government's Confucian conception is very different from the neo-liberal model where the government should be minimized. In Chinese philosophies, the government, at its best, 
is considered the source of moral guidance and moral influence (Whitman, 1985). Recently, with respects to the new developments of big data and big tech companies, we have seen the Chinese state takes on a seemingly paradoxical position, at least to the Western perspectives: it serves to guard against any intrusive measures of big tech companies' methods of data collection, simultaneously, collects a massive amount of data with little to no regards for any notion privacy (Roberts, Cowls, Morley, Taddeo, Wang, \& Floridi, 2020). This kind of Confucian-stateexceptionalism grants the Chinese state many leeways to wield its power in intervening with the lives of its citizens and companies.

From an acculturation perspective, these Confucian core values and norms regarding the individual and collective role are coming into a clash with Western privacy norms. One example is the Social Credit System (SCS), which further provides a juxtaposition of these two sets of values. Although the SCS— "a panopticon with Chinese characteristics" (Aho \& Duffield; 2020) receives a lot of bad press in Western media, legitimate studies in China reveal the Chinese public, on average, seems not to perceive this system negatively (Roberts et al., 2020). Whether on balance, the SCS is a good or bad thing requires long-term and careful research.

To researchers studying such sensitive issue, it is necessary to understand how ethical norms and values about privacy are changing during the process of acculturation. This is an interdisciplinary research, which requires insights from philosophy, sociology, culture and media studies, legal studies, to name a few. Since China could easily be seen as an aggressor country, it is a must to stick with the science, and remain open and humble to welcome contradicting insights (Vuong, 2018; 2020).

\section{References}


Aho, B., \& Duffield, R. (2020). Beyond surveillance capitalism: Privacy, regulation and big data in Europe and China. Economy and Society, 49(2), 187-212.

Han, D. (2018). Search boundaries: human flesh search, privacy law, and internet regulation in China. Asian Journal of Communication, 28(4), 434-447.

Liebman, B. L. (2011). The media and the courts: towards competitive supervision? The China Quarterly, 833-850.

Ma, Y. (2019). Unmapped privacy expectations in China: Discussions based on the proposed Social Credit System. Paper presented at the Information in Contemporary Society. iConference 2019. Lecture Notes in Computer Science, vol 11420., Cham.

Roberts, H., Cowls, J., Morley, J., Taddeo, M., Wang, V., \& Floridi, L. (2020). The Chinese approach to artificial intelligence: an analysis of policy, ethics, and regulation. AI \& SOCIETY. doi:10.1007/s00146-020-00992-2

Vuong, Q. H., \& Napier, N. K. (2015). Acculturation and global mindsponge: an emerging market perspective. International Journal of Intercultural Relations, 49, 354-367.

Vuong, Q.-H. (2018). The (ir)rational consideration of the cost of science in transition economies. Nature Human Behaviour, 2(1), 5-5. doi:10.1038/s41562-017-0281-4

Vuong, Q.-H., Bui, Q.-K., La, V.-P., et al. (2018). Cultural additivity: behavioural insights from the interaction of Confucianism, Buddhism and Taoism in folktales. Palgrave Communications, 4(1), 143. doi:10.1057/s41599-018-0189-2

Vuong, Q. H. (2020). Reform retractions to make them more transparent. Nature, 582(149). doi:https://doi.org/10.1038/d41586-020-01694-x 
Vuong, Q.-H., Ho, M.-T., Nguyen, H.-K. T., et al. (2020). On how religions could accidentally incite lies and violence: folktales as a cultural transmitter. Palgrave Communications, 6(1), 82. doi:10.1057/s41599-020-0442-3

Wang, Y., Balnaves, M., \& Sandner, J. (2020). Shameful secrets and self-presentation: Negotiating privacy practices among youth and rural women in china. SAGE Open, 10(1), 2158244020903396. doi:10.1177/2158244020903396

Wang, Z., \& Yu, Q. (2015). Privacy trust crisis of personal data in China in the era of Big Data: The survey and countermeasures. Computer Law \& Security Review, 31(6), 782-792. 